\title{
Down-Regulation of Renal Gluconeogenesis in Type II Diabetic Rats Following Roux-en-Y Gastric Bypass Surgery: A Potential Mechanism in Hypoglycemic Effect
}

\author{
Yi Wen Ning Lin Hong-Tao Yan Hao Luo Guang-Yu Chen \\ Jian-Feng Cui Li Shi Tao Chen TaoWang Li-Jun Tang \\ Department of General Surgery, Chengdu Military General Hospital, Chengdu, China
}

\section{Key Words}

Type 2 diabetes mellitus · Gluconeogenesis · Gastric bypass surgery · Insulin · Adiponectin

\begin{abstract}
Objective: This study was initiated to evaluate the effects of Roux-en-Y gastric bypass surgery on renal gluconeogenesis in type 2 diabetic rats and its relationship with hormonal parameters. Methods: Diabetic rats were induced by intraperitoneal injection of streptozotocin (STZ; $35 \mathrm{mg} / \mathrm{kg}$ ) combined with a high-fat diet. They were then randomly divided into three groups: diabetes model group (DM group, $n=8$ ), sham Roux-en-Y gastric bypass group (SRYGB group, $n=8$ ), and Roux-en-Y gastric bypass group (RYGB group, $n=14$ ). Another 8 normal rats comprised the normal control group (NC group, $\mathrm{n}=8$ ). Body weight, glucose, serum lipid, insulin, glucagon-like peptide-1 (GLP-1), leptin, and adiponectin were measured pre- and postoperatively. Glucose-6-phosphatase (G6Pase), phosphoenolpyruvate carboxykinase (PEPCK), insulin receptor- $\alpha$ (IR- $\alpha)$, insulin receptor- $\beta$ (IR- $\beta$ ), and glycogen synthase kinase 3 beta (Gsk3b) were measured in renal cortex by using RT-PCR and Western immune-blot analyses on the 4th week after operation. Results: Following RYGB surgery, surgery-treated rats showed significantly improved oral glucose tolerance, dyslipidemia and insulin resistance as well as increased post-gavage insulin levels and serum circulating levels of GLP-1 and adiponectin. RTPCR and Western immune-blot analyses showed PEPCK and G6Pase protein and mRNA to be significantly decreased in the renal cortex in the RYGB group ( $p<0.05$ vs. DM or SRYGB group); in addition, IR- $\alpha$ and Gsk3b phosphorylation levels increased in the RYGB group ( $<0.05$ vs. DM or SRYGB group). Conclusion: Down-regulation of renal gluconeogenic enzymes might be a potential mechanism in hypoglycemia. An improved insulin signal pathway in the renal cortex and increased circulating adiponectin concentrations may contribute to the decline of renal gluconeogenesis following RYGB surgery.


Wen et al.: Down-Regulation of Renal Gluconeogenesis in Type II Diabetic Rats Following Roux-en-Y Gastric Bypass Surgery: A Potential Mechanism in Hypoglycemic Effect

\section{Introduction}

Today, diabetes is a common endocrine disease; more than $90 \%$ of cases are type 2 diabetes mellitus (T2DM). In recent years, as changes in lifestyle and population aging accelerate, the prevalence of diabetes is rising rapidly all over the world, and more than $60 \%$ of T2DM patients are morbidly obese [1]. According to a report by the International Diabetes Federation (IDF), about 360 million people around the world had diabetes in 2011, and that number is expected to reach 520 million by 2030 [2]. T2DM has become one of the major chronic diseases threatening human health; therefore, effective prevention and treatment of T2DM is a chief public health issue.

Current management of T2DM is focused on the prevention of disease progression through lifestyle modifications and medical treatment. Although there have been tremendous advances in pharmacological treatment, in many cases these measures cannot adequately control diabetes [3] or prevent diabetes complications. Today, it is known that bariatric surgery, namely Roux-en-Y gastric bypass surgery (RYGB), results in unexpected therapeutic efforts in patients with metabolic syndrome and T2DM. It can produce sustained substantial weight loss and long-term treatment effects for T2DM. A meta-analysis demonstrated that following bariatric surgery T2DM was completely cured in $76.8 \%$ and alleviated in $86 \%$ of the patients [4]. Additionally, bariatric surgery results in benefits of other obesity-related comorbidities, such as dyslipidemia and obstructive sleep apnea, and improves health-related quality of life [4]. Several studies demonstrated reduced mortality due to cardiovascular disease, cancer in women, and T2DM itself $[5,6]$.

Several mechanisms have been proposed to explain improved glucose homeostasis post RYGB, and multiple changes including physical changes in the gut and hormone changes are responsible for the beneficial effects. In addition to the direct beneficial effects brought about by the reduction of calorie intake and weight loss, the changes in gastrointestinal hormones caused by gastrointestinal tract anatomic reconstruction after gastric bypass surgery play an important role in curing or improving T2DM [7]. Previous studies confirmed that gastric bypass surgery can increase insulin sensitivity and reactivity on insulin target organs, such as skeletal muscle, liver and fat tissue, improve the function of glucose metabolism in those organs, increase the use and storage of glucose, and reduce the production of endogenous glucose thus declining fasting and postprandial blood glucose levels [8].

Recent studies showed that the kidneys are involved in regulating glucose homeostasis inside the human body; besides the liver, the renal cortex also contains abundant enzymes related to gluconeogenesis that under various physiological conditions can produce glucose via gluconeogenesis and release it into the blood circulation [9]. In in patients suffering from diabetes, the expression of insulin receptors in the kidney has changed like that other insulin target organs [10], and the output of renal glucose is increased [9]. The increased production of renal glucose may be a contributing factor in the development of T2DM.

Therefore, alterations in renal gluconeogenesis represent a potentially important mechanism for regulating glucose homeostasis as well as its putative effects on the production of endogenous glucose after RYGB. This study was initiated to evaluate how RYGB affects renal gluconeogenesis in T2DM rats and how these effects are related to hormonal parameters, in order to determine the role of renal gluconeogenesis in glucose homeostasis after RYGB. 
Wen et al.: Down-Regulation of Renal Gluconeogenesis in Type II Diabetic Rats Following Roux-en-Y Gastric Bypass Surgery: A Potential Mechanism in Hypoglycemic Effect

\section{Material and Methods}

Animal and Diets

Male Sprague Dawley rats (180 $\pm 10 \mathrm{~g})$ were used in this study. The animals were purchased from Dashuo Animal Science and Technology Co., Ltd. (Chengdu, China). The animals were housed in standard cages and maintained under controlled room temperature $\left(22 \pm 2{ }^{\circ} \mathrm{C}\right)$ and humidity with a $12 / 12$-hour lightdark cycle. Rats had ad libitum access to water and rodent chow. Experimental procedures were approved by the Institutional Animal Care and Use Committee at the Chengdu Military General Hospital. The animals were maintained on a commercial rat feed manufactured by Dashuo Animal Science and Technology Co., Ltd., Chengdu, China. The high-fat diet consisted of $15 \%$ lard, $20 \%$ sucrose, $1 \%$ cholesterol, and $64 \%$ standard rat feed [11].

\section{Induction of an Animal Model}

40 male Sprague Dawley rats were randomly divided into the normal control group (NC group, $\mathrm{n}=8$ ) and diabetes modeling animal group $(\mathrm{n}=32$ ). The NC animal group consumed a standard diet, while the diabetes modeling animal group consumed a high-fat diet. After 4 weeks (week 5) the diabetes modeling animal were kept on an overnight fast and then injected once intraperitoneally with streptozotocin (Sigma St. Louis, MO, USA) at a dose of $35 \mathrm{mg} / \mathrm{kg}$ bw (STZ was dissolved in $0.1 \mathrm{~mol} / \mathrm{l}$ sodium citrate buffer $\mathrm{pH} 4.4$ and injected immediately to avoid degradation). After 1 week (week 6), oral glucose tolerance test (OGTT) was carried out. After a 12-hour fast, each rat was administered $1.5 \mathrm{~g}$ glucose/kg bw with a $50 \%$ solution by intragastric tube, and blood was taken from the tail vein; rats with blood glucose level $\geq 11.1 \mathrm{mmol} / \mathrm{l}$ at 120 min were considered to be diabetic and were used for the study. 30 rats showed diabetic modeling, and the modeling rate was $93.8 \%$. After 1 week (week 7) being stable, the modeling animals were randomly divided into the diabetes modeling group (DM group, $n=8$ ), the sham Roux-en-Y gastric bypass group (SRYGB group, $n=8$ ), and the Roux-en-Y gastric bypass group (RYGB group, $n=14$ ). The SRYGB group received transection and re-anastomosis of the small bowel. The RYGB group received the gastric bypass surgery, while the NC and DM groups did not receive surgical intervention. Throughout the study, both STZ-injected and non-injected animals continued on their original diet. The authors declared that the experiments conducted during these studies comply with the current laws of their country.

\section{Surgical Procedures and Animal Care}

A detailed description of the surgical technique, surgical controls, and perioperative care for RYGB has been published elsewhere. The day before surgery, rats fasted overnight but had free access to water. After randomization, rats were weighed and then anesthetized with $5 \%$ pentobarbital sodium solution (Sigma) at a dose of $50 \mathrm{mg} / \mathrm{kg}$ bw; ceftriaxone $100 \mathrm{mg} / \mathrm{kg}$ was given intramuscularly as a prophylactic antibiotic. Under sterile conditions, a midline laparotomy with a $3 \mathrm{~cm}$ incision was performed. Rats in the SRYGB group received transection and re-anastomosis of the small bowel. In the RYGB group, the distal stomach was closed through double ligation with 2-0 silk yarn to create a 20\% gastric pouch. The jejunum was cut off about 10 $\mathrm{cm}$ from the Tretiz ligaments. The proximal part of the stomach was opened and anastomosed with the distal jejunum. The proximal jejunum was anastomosed with the side of distal jejunum at the site about $10 \mathrm{~cm}$ away from the above stomach jejunum anastomosis. The gastrojejunostomies and jejunojejunostomies were performed using interrupted 5-0 silk sutures. After finishing the anastomosis, the abdomen was washed with $5 \mathrm{ml}$ metronidazole solution washing abdomen, followed by abdominal closure using 3-0 silk and 5-0 prolene. Surgical incisions were injected with $0.5 \mathrm{ml}$ of $0.25 \%$ bupivicaine to minimize postoperative discomfort. All rats were injected subcutaneously with normal saline (50 ml/kg before the start of surgery, immediately after surgery and again on postoperative day (POD) 1). After surgery, animals were housed individually, and body weight and food consumption were monitored daily. To allow the surgical anastamoses to heal, animals were not allowed to eat or drink for $24 \mathrm{~h}$ after surgery. Approximately $24 \mathrm{~h}$ after surgery, animals were started on a liquid diet consisting of resource and access to water ad libitum. Regular chow was started on POD 4 to ensure adequate healing of the stomach and bowel anastamoses. Rats in the SRYGB group were given the same amount of food as the RYGB rats [12].

OGTTS) and Insulin Sensitivity

OGTTs were performed preoperatively and repeated in postoperative weeks 2 and 4 . Before the tests, the rats fasted overnight; glucose $1.5 \mathrm{~g} / \mathrm{kg}$ bw (dissolved as a $50 \%$ solution) was administered by intragastric tube, and a drop of blood was taken from the tail vein at 0, 30, 60 and 120 min for measurement of blood 
Wen et al.: Down-Regulation of Renal Gluconeogenesis in Type II Diabetic Rats

Following Roux-en-Y Gastric Bypass Surgery: A Potential Mechanism in Hypoglycemic Effect

Table 1. Sequences and conditions of primers in used in PCR

\begin{tabular}{|c|c|c|c|c|}
\hline Gene & Acc. number & Primer sequences & $\begin{array}{l}\text { Amplification } \\
\text { length, bp }\end{array}$ & $\begin{array}{l}\text { Annealing } \\
\text { temperature, }{ }^{\circ} \mathrm{C}\end{array}$ \\
\hline PEPCK & NM_198780.3 & $\begin{array}{ll}\text { forward } & 5^{\prime} \text {-GCCTGGGGGACGTCAACGTG-3' } \\
\text { reverse } & 5^{\prime} \text {-CCTAGCTCCCAACGCGCGTGCC-3' }\end{array}$ & 247 & 60 \\
\hline G6Pase & NM_176077.3 & $\begin{array}{ll}\text { forward } & 5^{\prime} \text {-CCACTCTGAGCGCCGGCATC-3' } \\
\text { reverse } & 5^{\prime} \text {-AGGCGTAGTAGACCGCGGGG-3' }\end{array}$ & 171 & 60 \\
\hline Beta-actin & NM_031144.3 & $\begin{array}{ll}\text { forward } & 5^{\prime} \text {-ACGGTCAGGTCATCACTATCG-3' } \\
\text { reverse } & 5^{\prime} \text {-GGCATAGAGGTCTTTACGGATG-3' }\end{array}$ & 619 & 60 \\
\hline
\end{tabular}

glucose levels. Blood glucose was measured with a portable glucometer (ONETOUCH UltraEasy; LifeScan Inc., Wayne, PA, USA). The determined glucose values at the specified time points were used for calculation of the area under the curve $\left(\mathrm{AUC}_{0-120}\right)$ using the trapezoidal method according to the equation of $\mathrm{AUC}=0.25 \times(0$ $\mathrm{min}$ blood glucose value $+4 \times 30 \mathrm{~min}$ blood glucose value $+3 \times 120 \mathrm{~min}$ blood glucose value). Insulin determinations were made using serum from blood extracted from the angular vein, and insulin was measured by a rat-specific radioimmunoassay kit (Beijing FuRui Biotech, Co., Ltd., Beijing, China) according to the manufacturer's guidelines. Insulin sensitivity of the experimental animals was evaluated using the homeostasis model assessment - insulin resistence (HOMA-IR), HOMA-IR = fasting glucose $\times$ fasting insulin $/ 22.5$.

\section{Biochemical Analysis}

After the rats underwent overnight fasting, blood samples were withdrawn from the angular vein (under mild ether anesthesia) into plastic tubes with appropriate procoagulant and then allowed to clot for $30 \mathrm{~min}$, and serum was separated by centrifugation at $3,000 \mathrm{rpm}$ for $10 \mathrm{~min}$ at $4^{\circ} \mathrm{C}$. An appropriate number of aliquots were separated and stored at $-80^{\circ} \mathrm{C}$ until assaying. Triglycerides (TG), total cholesterol (TC), and free fatty acid (FFA) were measured by colorimetric assays using a commercially available kit (Nanjing Jiancheng Bioengineering Institute, Nanjing, Jiangsu, China) in accordance with the manufacturer's instructions. Rat-specific ELISA kits were used for determination of serum adiponectin, leptin (Shanghai KaiBo Biotech, Co., Ltd., Shanghai, China) and GLP-1 (Shanghai HaLing Biotech, Co., Ltd., Shanghai, China) concentrations.

\section{Tissue Collection}

All rats were sacrificed on POD 28. The rats were anesthetized with an intraperitoneal injection of 5\% pentobarbital sodium solution ( $50 \mathrm{mg} / \mathrm{kg}$ bw). Right and left kidneys were collected, weighed, and stored at $-80^{\circ} \mathrm{C}$ until further analysis.

\section{Fluorescent Quantitative Real-Time PCR}

Total RNA was extracted from renal cortical tissue by using the Trizol reagent (Beyotime Institute of Biotechnology, Beijing, China), which is an RNA extraction kit, in accordance with the manufacturer's instructions. RNA purity and concentration were determined using the Thermo NanoDrop-2000 spectrophotometer (Thermo Fisher Scientific Inc., Waltham, MA, USA) at A260/A280 nm. The purity of RNA obtained was 1.8-2.0. The primers were synthesized by Sangon Biotech Co., Ltd. (Shanghai, China) and are shown in table 1. Reverse transcription of RNA and PCR amplifications were performed with One Step SYBR ${ }^{\circledR}$ PrimeScript ${ }^{\text {TM }}$ RT-PCR Kit II (Takara Biotechnology (Dalian) Co., Ltd., Dalian, Liaoning, China) by C1000 ${ }^{\mathrm{TM}} \mathrm{Thermal}$ Cycler (Bio-Rad Inc., Hercules, CA, USA). The cycling program was as follows: $5 \mathrm{~min}$ at $42{ }^{\circ} \mathrm{C}, 10 \mathrm{~s}$ at $95^{\circ} \mathrm{C}$, followed by 40 cycles of $5 \mathrm{~s}$ at $95^{\circ} \mathrm{C}$ for and $30 \mathrm{~s}$ at $60^{\circ} \mathrm{C}$ and then dissociation. The reactions were quantified according to the amplification cycles when the PCR products of interest were first detected (threshold cycle, Ct). Each reaction was performed in triplicate. The expression of the transcripts was normalized to the levels of betaactin in the samples. Data was analyzed using CFX Manager ${ }^{\mathrm{TM}}$ Software 1.6 (Bio-Rad Inc.).

Western Blot Analysis

The proteins from the renal cortical tissue were extracted using a protein extraction kit (Nanjing KeyGen Biotech, Co., Ltd., Nanjing, Jiangsu, China). The protein concentrations were determined using an enhanced BCA Protein Assay kit (Nanjing KeyGen Biotech, Co., Ltd., Nanjing, Jiangsu, China). Extracted protein was then 
Fig. 1. Changes in body weight before and after the operation in NC $(n=8), D M(n=8)$, SRYGB ( $n=$ 8) and RYGB $(n=8)$ groups. ${ }^{*} p<$ 0.05 compared with normal control group; ${ }^{*} \mathrm{p}<0.05$ compared with DM group and SRYGB group. Data are presented as mean \pm SD.

Wen et al.: Down-Regulation of Renal Gluconeogenesis in Type II Diabetic Rats Following Roux-en-Y Gastric Bypass Surgery: A Potential Mechanism in Hypoglycemic Effect

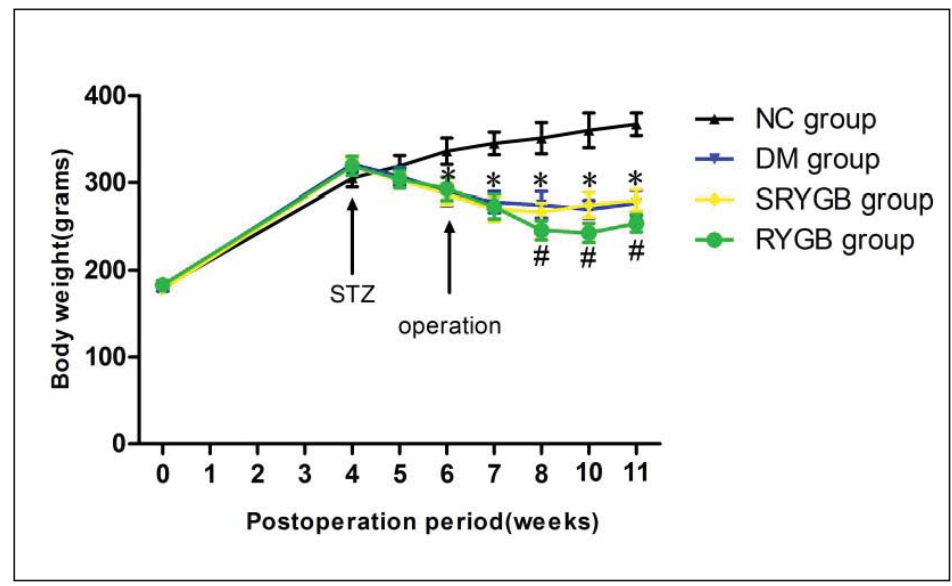

subjected onto $10 \%$ sodium dodecyl sulfate polyacrylamide gels. The electrophoresis time was dependent on the molecular weight of the protein. The separated proteins were transferred to polyvinylidene fluoride membranes (Merck, Darmstadt, Germany) by electrotransfer. The membranes were blocked with Trisbuffered saline with $0.05 \%$ Tween-20 (TBS-T) containing 5\% non-fat powdered milk for $1 \mathrm{~h}$ and then incubated with anti-PEPCK, anti-G6Pase, anti-IR- $\alpha$, anti-IR- $\beta$, anti-Gsk3b, and anti-phosphorylated Gsk3b (Ser 9) antibodies (Santa CruzBotechnology Inc., Dallas, TX, USA) in 5\% milk/TBS-T overnight. The membranes were rinsed three times with TBS-T and rinsed once with TBS and incubated with horseradish peroxidaseconjugated goat anti-rabbit antibody (diluted 1:1,000; Beyotime Institute of Biotechnology, Beijing, China) for $1 \mathrm{~h}$. Then the membranes were rinsed three times with TBS-T and rinsed once with TBS. Chemiluminescence detection reagent (Millipore, Billerica, MA, USA) was added drop-wise onto the membranes. The luminescent signal was detected by exposure to X-ray film.

\section{Statistical Analysis}

All data are presented as mean \pm SD and analyzed using SPSS 17 statistical software (SPSS, Inc., Chicago, IL, USA). Comparisons between groups were determined by one-way analysis of variance, and $\mathrm{p}<0.05$ was recognized as statistically significant.

\section{Results}

\section{Survival}

Among the 14 animals in the RYGB group, 8 survived for 4 weeks; the main reason for death was gastrointestinal anastomotic fistula and bowel obstruction. The most significant morbidity was noted within the first $48 \mathrm{~h}$ after surgery, and animals were euthanized if clinical criteria of decompensation were met. No animals died in the SRYGB group. Our final survival count used in the analysis to follow was NC $(n=8)$, DM $(n=8)$, SRYGB $(n=8)$, RYGB $(\mathrm{n}=8)$.

\section{Weight}

Changes in body weight over time for the different experimental groups are shown in figure 1 . At the baseline there was no difference in weight between the groups (180 $\pm 10 \mathrm{~g})$. Four weeks after feeding with a high-fat diet, the mean body weights of the NC (305 $\pm 10 \mathrm{~g})$, DM (321 \pm 9 g), SRYGB (318 $\pm 10 \mathrm{~g})$ and RYGB (319 $\pm 11 \mathrm{~g}$ ) groups were similar, though the NC group was slightly lighter. The NC group gained weight continuously. Two weeks after intraperitoneal injection with streptozotocin, the body weight in the DM $(290 \pm 16$ g), SRYGB $(287 \pm 12 \mathrm{~g})$ and RYGB $(293 \pm 13 \mathrm{~g})$ groups showed significant decreases $(\mathrm{p}<0.05)$ compared to the NC group, and stabilized at week 7. After operation, a closer comparison of the DM, 
Wen et al.: Down-Regulation of Renal Gluconeogenesis in Type II Diabetic Rats

Following Roux-en-Y Gastric Bypass Surgery: A Potential Mechanism in Hypoglycemic Effect

Table 2. Changes in serum lipid metabolic parameters in each group of rats (mean $\pm S D, m m o l / l, n=8$ )

\begin{tabular}{|c|c|c|c|c|c|c|}
\hline & \multicolumn{2}{|l|}{ Tch } & \multicolumn{2}{|l|}{$\mathrm{TG}$} & \multicolumn{2}{|l|}{ FFA } \\
\hline & pre-operation & $\begin{array}{l}4 \text { weeks } \\
\text { post-operation }\end{array}$ & pre-operation & $\begin{array}{l}4 \text { weeks } \\
\text { post-operation }\end{array}$ & pre-operation & $\begin{array}{l}4 \text { weeks } \\
\text { post-operation }\end{array}$ \\
\hline $\mathrm{NC}$ & $1.19 \pm 0.17$ & $1.15 \pm 0.15$ & $0.73 \pm 0.14$ & $0.78 \pm 0.10$ & $0.57 \pm 0.07$ & $0.63 \pm 0.09$ \\
\hline DM & $2.17 \pm 0.31^{*}$ & $2.41 \pm 0.32^{*}$ & $1.36 \pm 0.24^{*}$ & $1.44 \pm 0.26^{*}$ & $1.04 \pm 0.17^{*}$ & $1.10 \pm 0.17^{*}$ \\
\hline SRYGB & $2.08 \pm 0.28^{*}$ & $2.37 \pm 0.35^{*}$ & $1.43 \pm 0.30^{*}$ & $1.34 \pm 0.21^{*}$ & $1.08 \pm 0.19 *$ & $1.12 \pm 0.14^{*}$ \\
\hline RYGB & $2.11 \pm 0.35^{*}$ & $1.23 \pm 0.29^{\#}$ & $1.39 \pm 0.27 *$ & $0.91 \pm 0.11^{\#}$ & $1.12 \pm 0.15^{*}$ & $0.84 \pm 0.07^{* \#}$ \\
\hline
\end{tabular}

Tch = Total cholesterol; TG = triglyceride; FFA =free fatty acid. $\mathrm{NC}=$ normal control; $\mathrm{DM}=$ diabetes modeling; $\mathrm{SRYGB}=$ sham Roux-en-Y gastric bypass; RYGB = Roux-en-Y gastric bypass. ${ }^{*} \mathrm{p}<0.05$ compared with normal control group. ${ }^{\#} \mathrm{p}<0.05$ compared with DM group and SRYGB group.

SRYGB and RYGB groups showed that the significant difference $(\mathrm{p}<0.05)$ in weight began at week 8 ( 2 weeks postoperatively) and continued for the duration of the remaining period of this study.

\section{Serum Lipid Metabolic Parameters}

The parameters of the lipid metabolism (TC, TG, and FFA) were significantly increased in the DM, SRYGB and RYGB groups compared with the NC group pre-operatively. As shown in table 2, at 4 weeks after surgery, the RYGB group showed significantly lower serum lipid metabolic parameters when compared to the DM and SRYGB groups $(\mathrm{p}<0.05)$. The serum levels of TC and TG in the RYGB group were closer to those of the NC group ( $p>0.05$ ), while the serum FFA level in the RYGB group was a little higher than that of the NC group reaching statistical difference $(\mathrm{p}<0.05)$.

\section{Glucose Tolerance}

To evaluate the effects of RYGB on glucose tolerance, OGTT was performed. Before surgical intervention, mean fasting blood glucose levels (mmol/l) were $12.5 \pm 1.2$ in the DM group,11.9 \pm 1.1 in the SRYGB group,12.1 \pm 1.0 in the RYGB group, and $4.4 \pm 0.8$ in the NC group (fig. 2A). Compared to that of the DM, SRYGB and RYGB groups, preoperative glucose tolerance was higher in the NC group following OGTT ( $<<0.05$ ) (fig. 2D). After surgical intervention, the RYGB group demonstrated lower blood glucose at all time points following OGTT compared to the DM and SRYGB groups ( $<<0.05$ ) (fig. 2B,C), and there were no apparent differences when compared with the NC group at all time points, except for 30 min postgavage (fig. 2B,C). Glucose tolerance in the RYGB group was significantly improved as indicated by a $50 \%$ reduction in the AUC for blood glucose (fig. 2D). These results suggest that RYGB efficiently improved glucose tolerance in rats.

\section{Insulin}

Circulating fasting and postprandial insulin levels reflect pancreatic $\beta$-cell function; this is why serum insulin levels were determined. There were no significant differences in fasting serum insulin levels among all groups preoperatively: $31.5 \pm 3.9 \mathrm{mIU} / \mathrm{l}$ in the DM group, 33.2 $\pm 3.4 \mathrm{mIU} / \mathrm{l}$ in the SRYGB group, $31.9 \pm 3.7 \mathrm{mIU} / \mathrm{l}$ in the RYGB group and $26.2 \pm 3.2 \mathrm{mIU} / \mathrm{l}$ in the NC group. Postgavage insulin levels in the DM, SRYGB and RYGB groups remained elevated and stable over time, whereas the 30-min postgavage insulin levels in the NC group (78.6 \pm $8.8 \mathrm{mIU} / \mathrm{l})$ were significantly higher than those in the other groups $(\mathrm{p}<0.05)$ (fig. $3 \mathrm{~A})$. Compared to the DM and SRYGB groups, the RYGB animals showed a reduction in basal insulin level postoperatively ( $\mathrm{p}<0.05$ ) (fig. 3B). On postoperative week 2, postgavage insulin levels 
Wen et al.: Down-Regulation of Renal Gluconeogenesis in Type II Diabetic Rats Following Roux-en-Y Gastric Bypass Surgery: A Potential Mechanism in Hypoglycemic Effect

Fig. 2. Glucose tolerance. Blood glucose was measured at $0,30,60$ and $120 \mathrm{~min}$ following administration of $1.5 \mathrm{~g} / \mathrm{kg}$ bw glucose gavage in NC $(n=8)$, DM $(n=8)$, SRYGB $(\mathrm{n}=8)$ and RYGB $(\mathrm{n}=8)$ groups. ${ }^{*} \mathrm{p}<0.05$ compared with the normal control group; ${ }^{\#} \mathrm{p}<$ 0.05 compared with the DM group and SRYGB group. Data are presented as mean \pm SD. Blood glucose was measured $\mathbf{A}$ preoperatively, B 2 weeks and C 4 weeks postoperatively. D Area under the curve (AUC) of glucose.

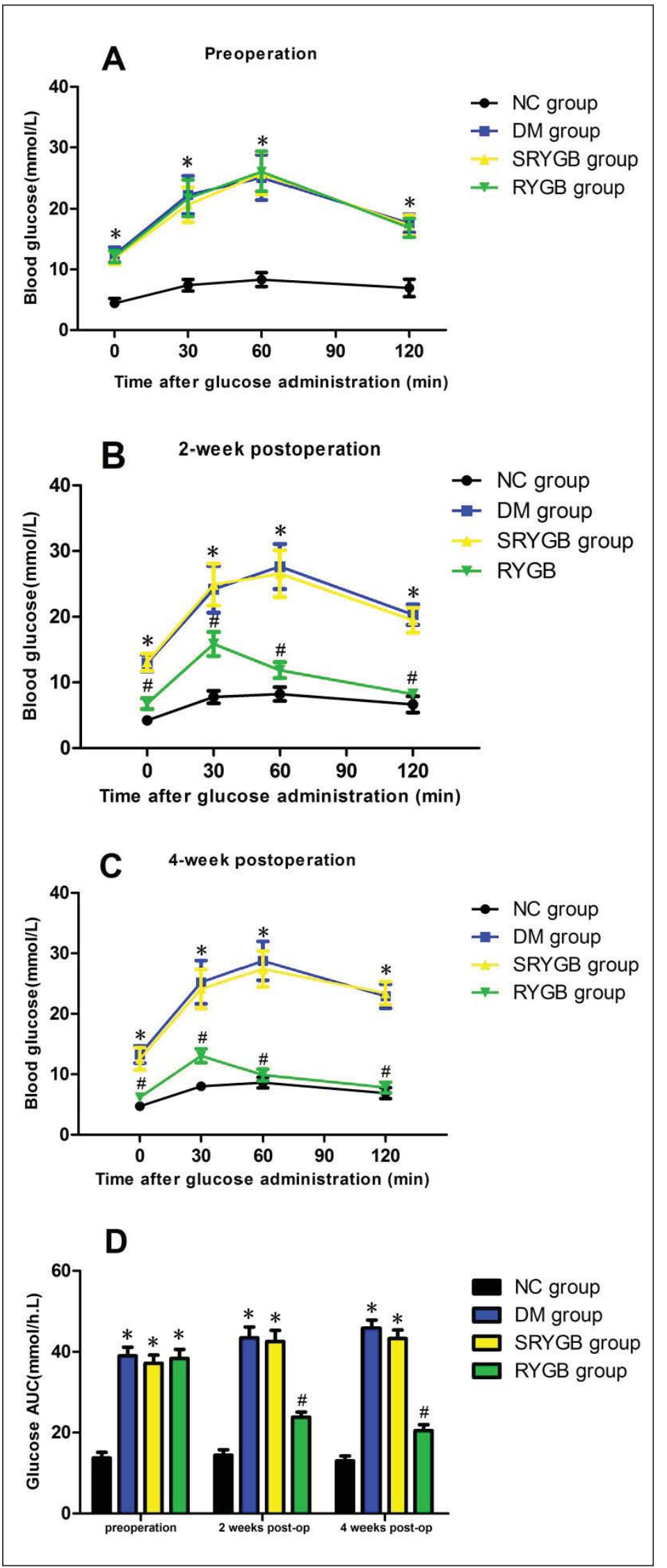


Wen et al.: Down-Regulation of Renal Gluconeogenesis in Type II Diabetic Rats Following Roux-en-Y Gastric Bypass Surgery: A Potential Mechanism in Hypoglycemic Effect

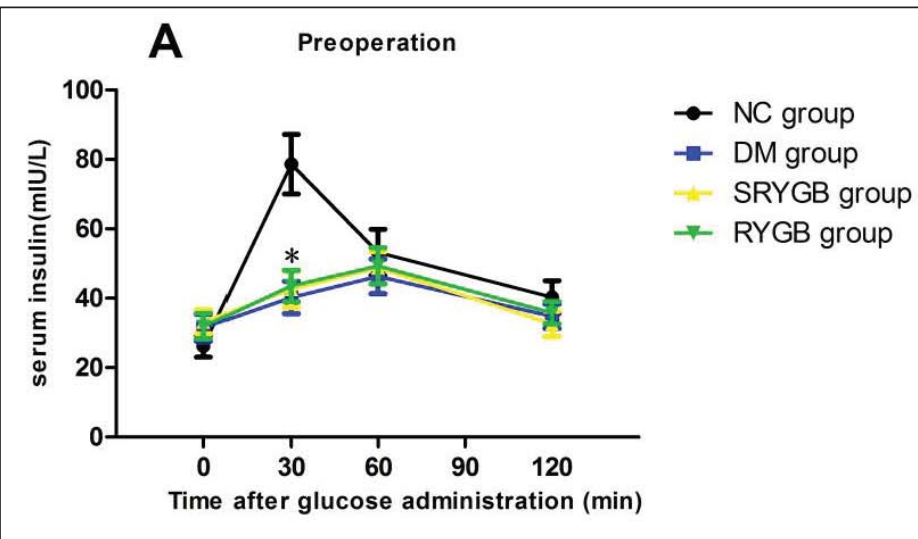

Fig. 3. Insulin level and HOMA. Serum insulin level was measured at 0, 30, 60 and $120 \mathrm{~min}$ following administration of $1.5 \mathrm{~g} / \mathrm{kg}$ bw glucose gavage in NC $(\mathrm{n}=8), \mathrm{DM}(\mathrm{n}=$ 8), SRYGB $(\mathrm{n}=8)$ and RYGB $(\mathrm{n}=$ 8) groups. *p $<0.05$ compared with the normal control group; ${ }^{\#} \mathrm{p}<0.05$ compared with the DM group and SRYGB group. Data are presented as mean \pm SD. Serum insulin was measured $\mathbf{A}$ preoperatively, B 2 weeks and C 4 weeks postoperatively. D HOMA-IR indexes.
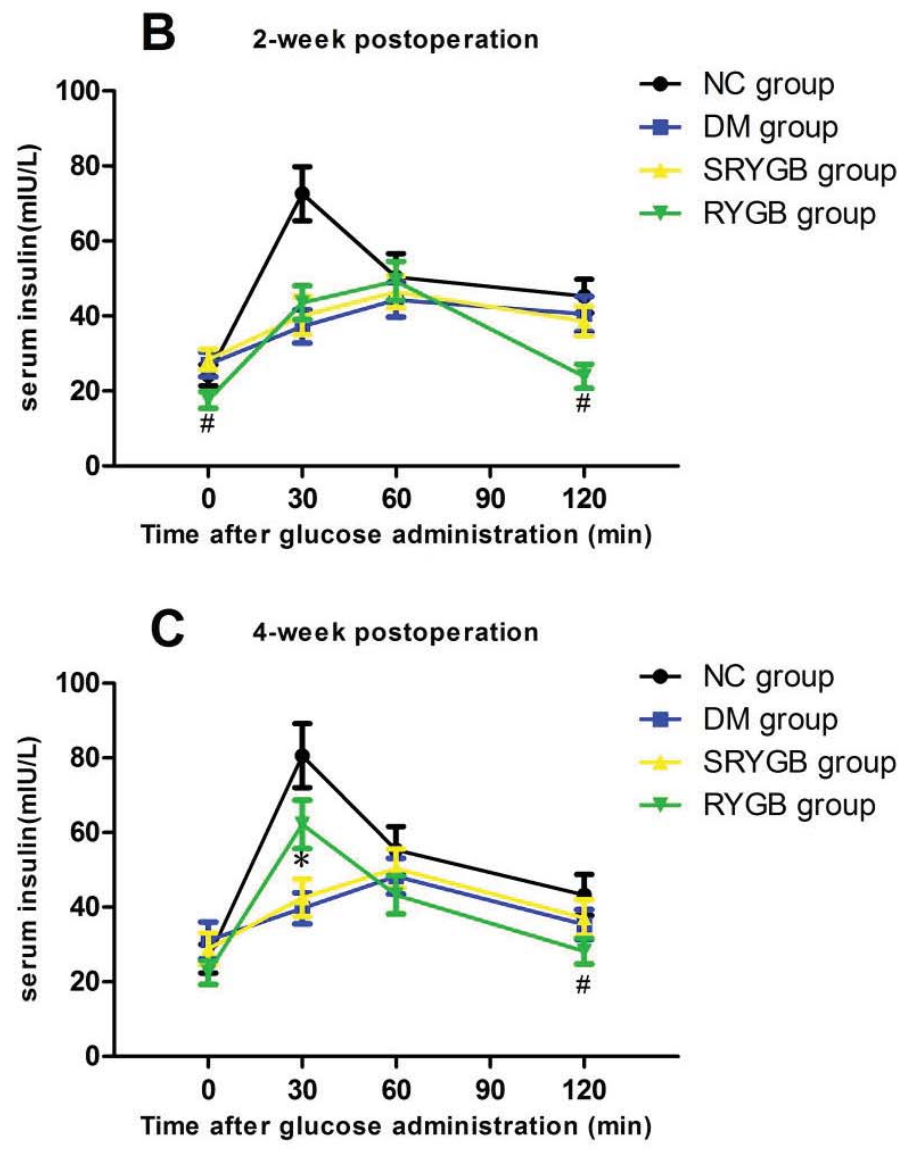

D

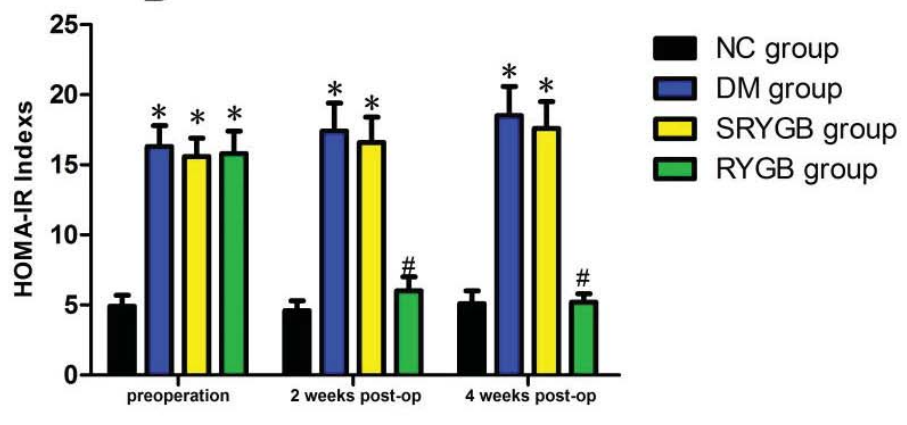


Wen et al.: Down-Regulation of Renal Gluconeogenesis in Type II Diabetic Rats

Following Roux-en-Y Gastric Bypass Surgery: A Potential Mechanism in Hypoglycemic Effect

Table 3. Changes in serum GLP-1, leptin and adiponectin in each group of rats (mean $\pm S D, n=8$ )

\begin{tabular}{|c|c|c|c|c|c|c|}
\hline & \multicolumn{2}{|l|}{ GLP-1, pmol/l } & \multicolumn{2}{|c|}{ Adiponectin, $\mu \mathrm{g} / \mathrm{ml}$} & \multicolumn{2}{|l|}{ Leptin, ng/ml } \\
\hline & pre-operation & $\begin{array}{l}4 \text { weeks } \\
\text { post-operation }\end{array}$ & pre-operation & $\begin{array}{l}4 \text { weeks } \\
\text { post-operation }\end{array}$ & pre-operation & $\begin{array}{l}4 \text { weeks } \\
\text { post-operation }\end{array}$ \\
\hline $\mathrm{NC}$ & $8.56 \pm 1.53$ & $9.87 \pm 1.89$ & $3.89 \pm 0.61$ & $3.78 \pm 0.69$ & $2.75 \pm 0.56$ & $2.86 \pm 0.61$ \\
\hline DM & $7.86 \pm 1.65$ & $8.13 \pm 2.1$ & $2.88 \pm 0.54^{*}$ & $1.91 \pm 0.34^{*}$ & $3.24 \pm 0.77$ & $3.45 \pm 0.78$ \\
\hline SRYGB & $7.92 \pm 1.32$ & $8.32 \pm 1.82$ & $2.67 \pm 0.48^{*}$ & $1.77 \pm 0.32^{*}$ & $3.36 \pm 0.72$ & $3.54 \pm 0.81$ \\
\hline RYGB & $8.1 \pm 1.54$ & $16.54 \pm 1.48^{* \#}$ & $2.75 \pm 0.41^{*}$ & $3.45 \pm 0.59^{\#}$ & $3.39 \pm 0.67$ & $3.17 \pm 0.62$ \\
\hline
\end{tabular}

GLP-1 = Glucagon-like peptide-1; NC = normal control; DM = diabetes modeling; SRYGB = sham Roux-en-Y gastric bypass; RYGB = Roux-en-Y gastric bypass. ${ }^{*} \mathrm{p}<0.05$ compared with normal control group. ${ }^{*} \mathrm{p}<0.05$ compared with DM group and SRYGB group.

Table 4. The expressions of PEPCK and G6Pase mRNA in each group (mean $\pm \mathrm{SD}, \mathrm{n}=8$ )

\begin{tabular}{lll}
\hline Groups & PEPCK & G6Pase \\
\hline Control & $1.0 \pm 0.09$ & $1.0 \pm 0.16$ \\
DM & $6.04 \pm 0.74^{*}$ & $4.26 \pm 0.66^{*}$ \\
SRYGB & $6.19 \pm 0.82^{*}$ & $4.45 \pm 0.69^{*}$ \\
RYGB & $3.68 \pm 0.38^{* \#}$ & $1.37 \pm 0.23^{\#}$ \\
\hline
\end{tabular}

PEPCK = Phosphoenolpyruvate carboxykinase; G6Pase = glucose6-phosphatase; $\mathrm{NC}=$ normal control; $\mathrm{DM}=$ diabetes modeling; SRYGB = sham Roux-en-Y gastric bypass; RYGB = Roux-en-Y gastric bypass.

${ }^{*} \mathrm{p}<0.05$ compared with normal control group.

${ }^{\#} \mathrm{p}<0.05$ compared with DM group and SRYGB group.

in the RYGB group showed no apparent changes while in the other groups there was a notable upward tendency (fig. 3B). On postoperative week 4, the insulin levels in the RYGB group were significantly higher than those of the DM or SRYGB groups at $30 \mathrm{~min}$, then decreased to basal levels from 60 to 120 min post gavage ( $<$ 0.05) (fig. 3C). The above results suggest that glucose-stimulated insulin response was increased in the RYGB group after surgery.

HOMA-IR

In this study, we used the HOMA-IR as an indicator of insulin sensitivity to confirm the hypothesis that RYGB improves insulin resistance in T2DM rats. As shown in fig. 3D, HOMA-IR values in diabetic animals were significantly higher than those in the NC group preoperatively $(p<0.05)$. HOMA-IR values in the postoperative RYGB group were significantly lower than those in the SRYGB and RYGB groups at the same time points as expected $(\mathrm{p}<0.05)$. On postoperative week 4, there were no significantly differences between the RYGB and NC groups. These results suggested that RYGB could improve insulin resistance in T2DM rats.

\section{Measurement of GLP-1, Leptin, and Adiponectin}

To determine the effect of gastric bypass surgery on hormones, the serum circulating levels of GLP-1, leptin, and adiponectin were detected. As shown in table 3, there was a decrease in GLP-1 and adiponectin in the DM and SRYGB groups compared with the NC group. Following RYGB surgery, the serum circulating levels of GLP-1 and adiponectin were significantly increased in the RYGB group compared with those in the DM and SRYGB groups. The serum circulating levels of leptin were not different between groups. These results suggest that the serum levels of several hormones were changed in response to RYGB surgery. 


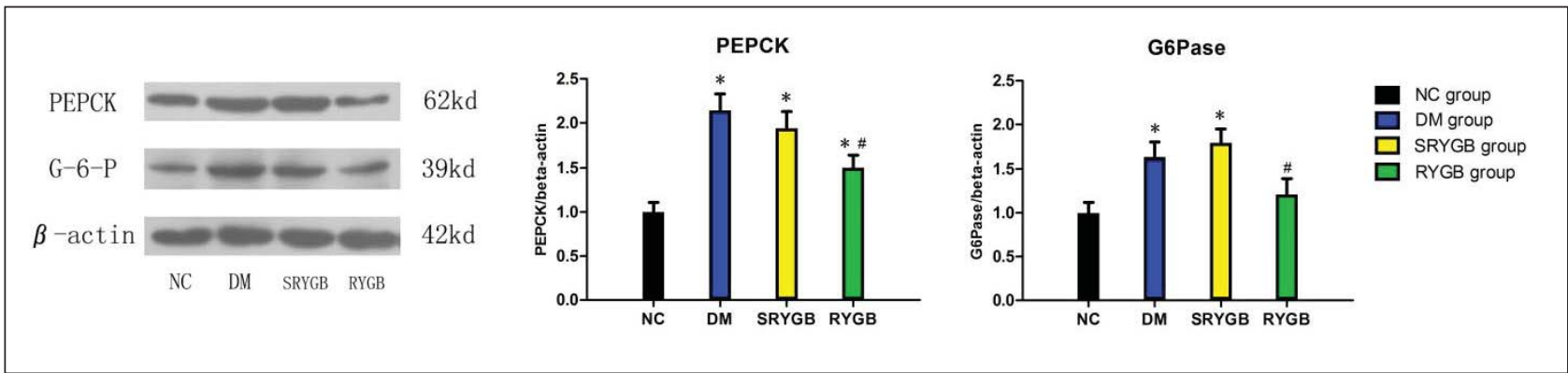

Fig. 4. Protein expressions of renal cortical G6Pase and PEPCK in NC $(n=8)$, DM $(n=8)$, SRYGB $(n=8)$ and RYGB ( $n=8)$ groups. A representative immunoblot for G6Pase and PEPCK is shown. ${ }^{*} \mathrm{p}<0.05$ compared with the normal control group; quantitative densitometric analyses of the amount of G6Pase and PEPCK normalized to beta-actin. ${ }^{\#}$ p $<0.05$ compared with the DM group and SRYGB group. Data are presented as mean \pm SD.

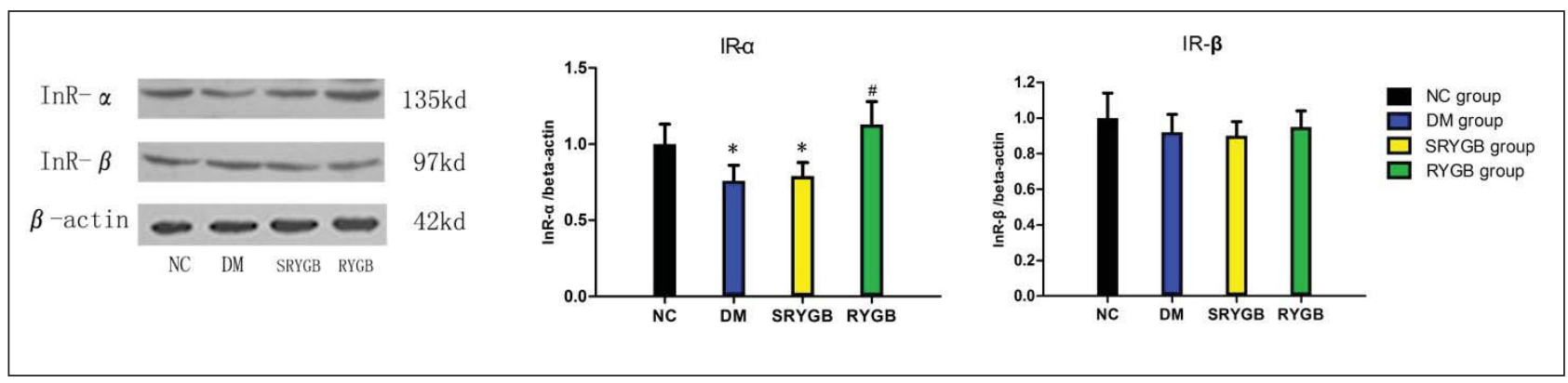

Fig. 5. Protein expressions of renal cortical IR- $\alpha$ and IR- $\beta$ in NC $(n=8)$, DM $(n=8)$, SRYGB $(n=8)$ and RYGB $(\mathrm{n}=8)$ groups. A representative immunoblot for IR- $\alpha$ and IR- $\beta$ is shown. ${ }^{*} \mathrm{p}<0.05$ compared with the normal control group; quantitative densitometric analyses of the amount of IR- $\alpha$ and IR- $\beta$ normalized to beta-actin. \# $\mathrm{p}<0.05$ compared with the DM group and SRYGB group. Data are presented as mean \pm SD.

\section{Expression of G6Pase and PEPCK}

To determine the effects of RYGB on renal gluconeogenesis, the mRNA and protein expressions of G6Pase and PEPCK in the renal cortex were investigated by RT-PCR and Western immunoblot analyses. As shown in table 4 and figure 4, the mRNA and protein expressions of renal cortical G6Pase and PEPCK in the DM and SRYGB groups were significantly higher than those in the NC group at the end of this study. Compared to the DM and SRYGB groups, the mRNA and protein expressions of renal cortical G6Pase and PEPCK in the RYGB group were decreased by some degrees. These results suggest that the renal gluconeogenesis was decreased following RYGB.

\section{Expression of IR- $\alpha$ and IR- $\beta$}

To determine whether the renal cortex exhibits insulin resistance in the diabetic state, the protein expressions of IR- $\alpha$ and IR- $\beta$ were investigated. As shown in figure 5 , the protein expressions of IR- $\alpha$ in the DM and SRYGB groups were significantly lower than those in the NC group at the end of this study. Compared to the DM and SRYGB groups, the protein expressions of IR- $\alpha$ in the RYGB group were apparently increased while the expression of IR- $\beta$ protein showed no difference between groups. 


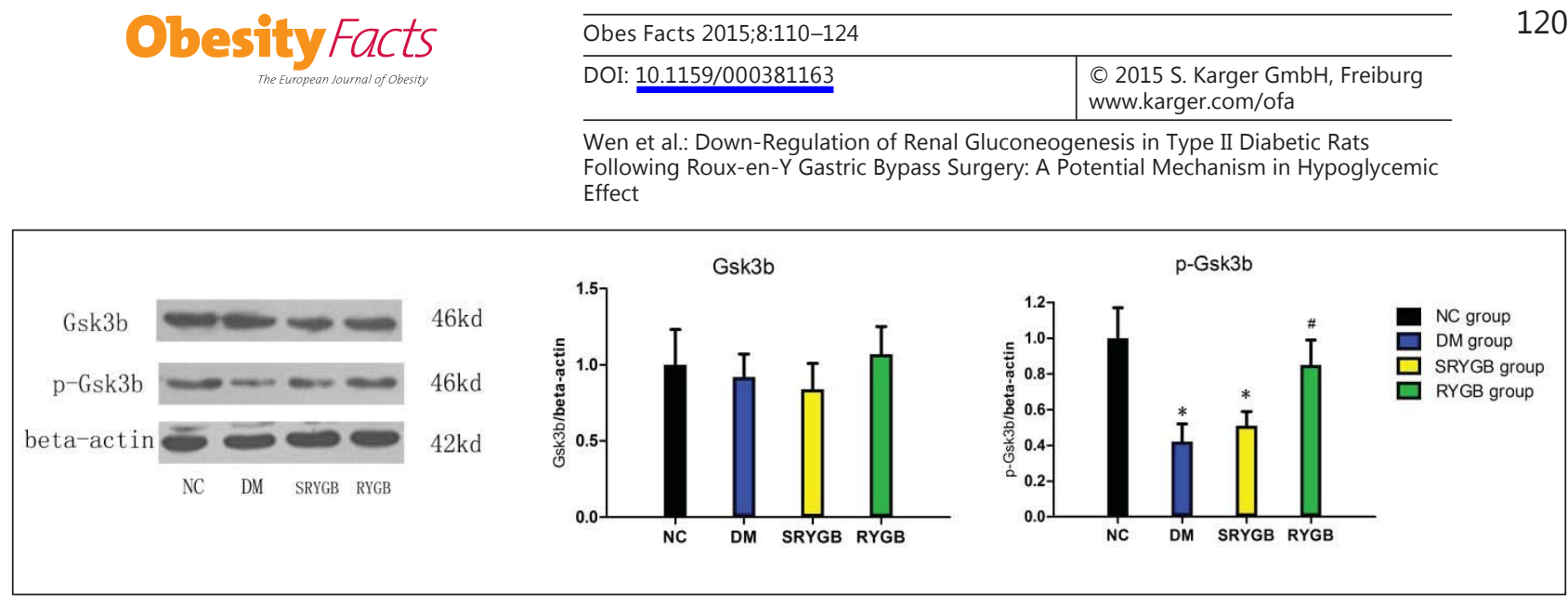

Fig. 6. Protein and phosphorylation expressions of renal cortical Gsk3b in NC ( $=8)$, DM ( $n=8)$, SRYGB $(\mathrm{n}=8)$ and RYGB $(\mathrm{n}=8)$ groups. A representative immunoblot for GSK3 is shown. ${ }^{*} \mathrm{p}<0.05$ compared with the normal control group; quantitative densitometric analyses of the amount of Gsk3b normalized to betaactin. ${ }^{\#} \mathrm{p}<0.05$ compared with the DM group and SRYGB group. Data are presented as mean \pm SD.

\section{Expression of Gsk3b and Phosphorylation}

To compare the activation of the insulin signaling cascade between the groups, Gsk3b phosphorylation and protein expressions were determined in the renal cortex. Gsk3b is a key signaling molecule in the insulin pathway; its phosphorylation levels are negatively correlated with activity. As shown in figure 6, the expression of Gsk3b showed no difference between groups. However, a reduction of Gsk3b phosphorylation was observed in the DM and SRYGB groups. Four weeks after RYGB surgery, Gsk3b phosphorylation levels in the RYGB group were significantly increased compared with those in the DM and SRYGB groups.

\section{Discussion}

T2DM is a disease that has become one of the major chronic diseases threatening human health. Although diet control can reduce about $5 \%$ of body mass and oral hypoglycemic drugs or insulin injection treatment allow for controlled blood glucose homeostasis in the short term, the long-term effect is not satisfactory nor has a cure been found. With bariatric surgery, namely RYGB surgery, unexpected therapeutic results in patients with metabolic syndrome and T2DM could be achieved; T2DM was completely cured in more than $80 \%$ of bariatric surgery patients [13]. Two prospective studies published in the New England Journal of Medicine [14, 15] assessed the curative efficacy and safety of bariatric surgery and drug therapy for obese patients with T2DM recently; the results these studies indicated that gastric bypass surgery is a safe and effective treatment of poor glycemic control in patients with T2DM and could improve blood glucose levels, reduce body weight and improve insulin resistance while also significantly reducing obesity and blood pressure and improving dyslipidemia, a risk factor for cardiovascular disease. In the present study, we confirmed that the RYGB group obtained sustained significant weight loss compared with the DM and SRYGB groups during the 28 -day study period, albeit less than the $30 \%$ reduction in total body weight commonly reported after RYGB in humans. Secondly, the levels of blood glucose and parameters of lipid metabolism in the RYGB group were significantly lower than those in the DM and SRYGB groups after surgery. These results demonstrated that gastric bypass surgery was suitable for the treatment of T2DM and associated dyslipidemia which is in accordance with the conclusions of previous clinical reports [7].

T2DM is recognized as insulin-independent diabetes; its pathogenesis is characterized by peripheral insulin resistance that can be accompanied by impaired insulin secretory 
Wen et al.: Down-Regulation of Renal Gluconeogenesis in Type II Diabetic Rats

Following Roux-en-Y Gastric Bypass Surgery: A Potential Mechanism in Hypoglycemic Effect

capacity of pancreatic $\beta$-cells. As a result of insulin resistance, the response of organs and tissues to insulin is weakened, thus causing a reduction in the utilization and storage of glucose and an increase in the production of endogenous glucose. Endogenous glucose is produced mainly via two pathways - gluconeogenesis and glycogenolysis - which are both enhanced in T2DM and contribute to fasting and postprandial hyperglycemia. The liver is regarded as the major gluconeogenesis organ, and most clinical discussions on glucose dysregulation focused on the liver as well as the intestine, pancreas, adipose tissue, and muscle $[16,17]$. More recently, the significance of the kidney for glucose homeostasis both under physiological and pathological conditions has become apparent. Besides the liver, the renal cortex has the highest expression of enzymes related to gluconeogenesis and is therefore capable of generating considerable amounts of glucose (gluconeogenesis) to be released into the circulation under various physiological conditions.

The results of this study confirmed that gastric bypass surgery is an effective treatment for T2DM. Following gastric bypass surgery, the RYGB group of rats showed improvement in insulin sensitivity and glucose tolerance, while the DM and SRYGB rats did not show a similar change. The data in our study also indicated that, compared to the DM and SRYGB groups, postgavage insulin levels increased gradually in the RYGB groups, suggesting $\beta$-cell survival and function. As gastric bypass surgery has been shown to improve glucose levels, we aimed to understand how glucose homeostasis is achieved and whether or not renal gluconeogenesis may play a significant role. According to previous research, the renal cortex contributes to approximately $20 \%$ of total body glucose release and is potentially responsible for approximately $40 \%$ of overall gluconeogenesis [18]. There is evidence that renal glucose release is increased in both the postprandial and the fasting state of patients with T2DM, and a threefold increase in renal glucose release was observed in patients with diabetes when compared to those without [19]. Taking into consideration that gluconeogenesis presumably accounts for approximately half of all circulatory glucose release during the fasting state, the kidneys appear to play an important role in overall glucose release in normal as well as pathophysiological states. PEPCK and G6Pase are the key rate-limiting enzymes in gluconeogenesis. In our study, overexpression of mRNA and protein of PEPCK and G6Pase was observed in the DM and SRYGB groups when compared with the NC group. Because our diabetic rats were not acidotic, a stimulation of gluconeogenesis in the renal cortex by metabolic acidosis can be ruled out. These findings are in accordance with previous studies [20] indicating that an increase of renal glucose delivery into the circulation is a major contributor to fasting hyperglycemia in diabetes. Following gastric bypass surgery, our data showed that PEPCK and G6Pase mRNA and protein levels in the renal cortex were significantly decreased in the RYGB group when compared with the DM and SRYGB groups. These changes in the RYGB group correlated with a decrease in glucose levels and an improvement in insulin resistance which both were obviously not different from those found in the NC group. In conclusion, we suggest that the expression changes of PEPCK and G6Pase may decrease renal glucose delivery, thus reducing endogenous glucose production and contributing to improved glucose homeostasis following RYGB surgery.

The down-regulating expression of key gluconeogenesis enzymes in the renal cortex after RYGB surgery is accompanied by an increase in postgavage insulin levels and improvement in insulin resistance. By down-regulating the expression of enzymes related to gluconeogenesis, insulin is the main negative modulator of renal gluconeogenesis [21]. Insulin action is dependent on the interaction with the insulin receptor. After binding to the receptor, insulin induces a downstream cascade of signaling molecules regulating the expression of enzymes involved in gluconeogenesis. Therefore, we evaluated the expression levels of IR and Gsk3b in the renal cortex. It could be shown that IR- $\alpha$ expression was significantly decreased in the DM and SRYGB groups compared with the NC group, while the expression of IR- $\beta$ was 
not different between groups. These results point to a renal insulin resistance present in our diabetic rat model. This is in accordance with results of Tiwari et al. [10]. In the RYGB group IR- $\alpha$ expression was increased postoperatively when compared to the DM and SRYGB groups. Furthermore, to compare the activation of the insulin signaling cascade between the groups, the expression and phosphorylation of a key signaling molecule of the insulin pathway, Gsk3b, in the renal cortex were determined. Gsk3b is a key kinase involved in dysregulated glucose metabolism and insulin resistance; its inactivation by insulin is considered essential for a normal insulin catabolic response [22]. Indeed, several studies have implicated dysregulation of GSK3 in the pathogenesis of diabetes, and Gsk3b positively regulates the expression of gluconeogenesis enzymes in the liver [23]. As the GSK3 inhibitor L803-mts down-regulates gene expression of gluconeogenesis enzymes in the liver and muscle of diabetic mice [24], we assumed that a Gsk3b-mediated mechanism is triggering overexpression of gluconeogenesis enzymes in the diabetic renal cortex too. We observed a significant alteration of Gsk3b phosphorylation levels between the NC and DM groups, suggesting that Gsk3b activity was enhanced in the renal cortex in the diabetic state, thus contributing to the overexpression of gluconeogenesis enzymes. In the RYGB group, the postoperative Gsk3b phosphorylation level in the renal cortex was significantly increased when compared with the DM and SRYGB groups. These results imply that the decline of renal gluconeogenesis following RYGB is related to an improved insulin signaling pathway.

Additionally, we also detected the effect of gastrointestinal bypass on hormones. There was an increase in GLP-1 and adiponectin and no significant difference in leptin between groups, but only adiponectin was directly associated with gluconeogenesis. Experimental studies showed that GLP-1 has extensive biological effects, e.g., increasing the synthesis and release of insulin, enhancing peripheral tissue insulin sensitivity and promoting regeneration in $\beta$-cells and islet cells, suppressing appetite, etc. [25]. Its effect on renal gluconeogenesis may be related to the impact on insulin action.

The increase in adiponectin after gastrojejunal bypass may also contribute to the renal gluconeogenesis change found postoperatively. The relationship between adiponectin and glucose homeostasis is complex and variable. The main action of adiponectin results from improved insulin resistance and an increase in muscle glucose transport and enhance energy consumption and fatty acid oxidation [26]. Significantly decreased plasma levels of adiponectin have been observed in obese/diabetic mice and humans [27]. In our study we also found that adiponectin levels in the DM and SRYGB group were significantly lower than those in the NC group. The insulin-sensitizing action is associated with circulating adiponectin concentration, which negatively correlates with endogenous glucose production in T2DM individuals [28]. The enhancement of hepatic insulin sensitivity by adiponectin leads to the down-regulation of the expression of key gluconeogenic genes, including G6Pase and PEPCK [29]. As renal gluconeogenesis and hepatic gluconeogenesis are similar physiological processes, we assume that the inhibition effect of adiponectin on the expression of gluconeogenic enzymes and the rate of endogenous glucose production may influence the process of renal gluconeogenesis.

Our study has several limitations. Although the relative abundance of PEPCK and G6Pase generally reflect their enzymatic activity, we did not measure their enzymatic activity in the present study. Secondly, we did not measure renal glucose production. Nevertheless, the increased expression of gluconeogenic enzymes in the renal cortex is associated with elevated plasma glucose, impaired glucose tolerance, and insulin resistance in our diabetic rat model. Despite its limitations, the present study provides important information with regard to the effects of diabetes and RYGB on renal gluconeogenesis.

In summary, our results suggest that gastric bypass surgery could decrease glucose and serum lipid levels, improve insulin resistance, and increase postgavage insulin levels in T2DM 


\begin{tabular}{l|l}
\hline \multicolumn{2}{l}{ Obes Facts 2015;8:110-124 } \\
\hline DOI: $10.1159 / 000381163$ & $\begin{array}{l}\text { C) 2015 S. Karger GmbH, Freiburg } \\
\text { www.karger.com/ofa }\end{array}$ \\
\hline
\end{tabular}

Wen et al.: Down-Regulation of Renal Gluconeogenesis in Type II Diabetic Rats Following Roux-en-Y Gastric Bypass Surgery: A Potential Mechanism in Hypoglycemic Effect

rats. Down-regulation of renal gluconeogenic enzymes may be a potentially important mechanism. Improved insulin signal pathway in the renal cortex and increased circulating adiponectin levels may contribute to the decline of renal gluconeogenesis following RYGB surgery.

\section{Disclosure Statement}

The authors declare that they have no conflicts of interest.

\section{References}

1 Kramer H, Cao G, Dugas L, Luke A, Cooper R, Durazo-Arvizu R: Increasing BMI and waist circumference and prevalence of obesity among adults with type 2 diabetes: the National Health and Nutrition Examination Surveys. J Diabetes Complications 2010;24:368-374.

-2 Whiting DR, Guariguata L, Weil C, Shaw J: IDF diabetes atlas: global estimates of the prevalence of diabetes for 2011 and 2030. Diabetes Res Clin Pract 2011;94:311-321.

-3 Nathan DM, Buse JB, Davidson MB, Ferrannini E, Holman RR, Sherwin R, Zinman B; American Diabetes Association; European Association for the Study of Diabetes: Medical management of hyperglycaemia in type 2 diabetes mellitus: a consensus algorithm for the initiation and adjustment of therapy: a consensus statement from the American Diabetes Association and the European Association for the Study of Diabetes. Diabetologia 2009;52:17-30.

4 Buchwald H, Avidor Y, Braunwald E, Jensen MD, Pories W, Fahrbach K, Schoelles K: Bariatric surgery: a systematic review and meta-analysis. JAMA 2004,292:1724-1737.

5 Adams TD, Gress RE, Smith SC, Halverson RC, Simper SC, Rosamond WD, Lamonte MJ, Stroup AM, Hunt SC: Long-term mortality after gastric bypass surgery. N Engl J Med 2007;357:753-761.

6 Adams TD, Hunt SC: Cancer and obesity: effect of bariatric surgery. World J Surg 2009;33:2028-2033.

7 Rubino F, Schauer PR, Kaplan LM, Cummings DE: Metabolic surgery to treat type 2 diabetes: clinical outcomes and mechanisms of action. Annual Rev Med 2010,61:393-411.

8 Dixon JB, le Roux CW, Rubino F, Zimmet P: Bariatric surgery for type 2 diabetes. Lancet 2012;379:2300-2311.

-9 Triplitt CL: Understanding the kidneys' role in blood glucose regulation. Am J Manag Care 2012,18(1 suppl):S11-16.

10 Tiwari S, Halagappa VK, Riazi S, Hu X, Ecelbarger CA: Reduced expression of insulin receptors in the kidneys of insulin-resistant rats. J Am Soc Nephrol 2007;18:2661-2671.

11 Srinivasan K, Viswanad B, Asrat L, Kaul CL, Ramarao P: Combination of high-fat diet-fed and low-dose streptozotocin-treated rat: a model for type 2 diabetes and pharmacological screening. Pharmacol Res 2005;52: 313-320.

12 Meirelles K, Ahmed T, Culnan DM, Lynch CJ, Lang CH, Cooney RN: Mechanisms of glucose homeostasis after Roux-en-Y gastric bypass surgery in the obese, insulin-resistant Zucker rat. Ann Surg 2009;249:277-285.

13 Yan H, Tang L, Chen T, Kral JG, Jiang L, Li Y, Hu R, Cui J, Li S, Tian F: Defining and predicting complete remission of type 2 diabetes: a short-term efficacy study of open gastric bypass. Obes Facts 2013;6:176-184.

14 Schauer PR, Kashyap SR, Wolski K, Brethauer SA, Kirwan JP, Pothier CE, Thomas S, Abood B, Nissen SE, Bhatt DL: Bariatric surgery versus intensive medical therapy in obese patients with diabetes. N Engl J Med 2012; 366:1567-1576.

15 Mingrone G, Panunzi S, De Gaetano A, Guidone C, Iaconelli A, Leccesi L, Nanni G, Pomp A, Castagneto M, Ghirlanda G, Rubino F: Bariatric surgery versus conventional medical therapy for type 2 diabetes. N Engl J Med 2012;366:1577-1585.

16 Meyer C, Woerle HJ, Dostou JM, Welle SL, Gerich JE: Abnormal renal, hepatic, and muscle glucose metabolism following glucose ingestion in type 2 diabetes. Am J Physiol Endocrinol Metab 2004;287:E1049-1056.

$\checkmark 17$ Wolff BS, Meirelles K, Meng Q, Pan M, Cooney RN: Roux-en-Y gastric bypass alters small intestine glutamine transport in the obese Zucker rat. Am J Physiol Gastrointest Liver Physiol 2009;297:G594-601.

18 Meyer C, Stumvoll M, Dostou J, Welle S, Haymond M, Gerich J: Renal substrate exchange and gluconeogenesis in normal postabsorptive humans. Am J Physiol 2002;282:E428-434.

19 Marsenic O: Glucose control by the kidney: an emerging target in diabetes. Am J Kidney Dis 2009;53:875-883.

20 Mitrakou A: Kidney: its impact on glucose homeostasis and hormonal regulation. Diabetes Res Clin Pract 2011; 93(suppl 1):S66-72.

21 DeFronzo RA, Davidson JA, Del Prato S: The role of the kidneys in glucose homeostasis: a new path towards normalizing glycaemia. Diabetes Obes Metab 2012;14:5-14.

22 Patel S, Doble BW, MacAulay K, Sinclair EM, Drucker DJ, Woodgett JR: Tissue-specific role of glycogen synthase kinase 3beta in glucose homeostasis and insulin action. Mol Cell Biol 2008;28:6314-6328.

23 Sakamaki J, Daitoku H, Kaneko Y, Hagiwara A, Ueno K, Fukamizu A: GSK3b regulates gluconeogenic gene expression through HNF4a and FOXO1. J Recept Signal Transduct Res 2012;32:96-101. 
Wen et al.: Down-Regulation of Renal Gluconeogenesis in Type II Diabetic Rats

Following Roux-en-Y Gastric Bypass Surgery: A Potential Mechanism in Hypoglycemic Effect

24 Kaidanovich-Beilin O, Eldar-Finkelman H: Long-term treatment with novel glycogen synthase kinase-3 inhibitor improves glucose homeostasis in ob/ob mice: molecular characterization in liver and muscle. J Pharmacol Exp Ther. 2006;316:17-24.

25 Girard J: The incretins: from the concept to their use in the treatment of type 2 diabetes. Part A: incretins: concept and physiological functions. Diabetes Metab 2008,34:550-559.

-26 Kadowaki T, Yamauchi T, Kubota N: The physiological and pathophysiological role of adiponectin and adiponectin receptors in the peripheral tissues and CNS. FEBS Lett 2008;582:74-80.

-27 Fang X, Sweeney G: Mechanisms regulating energy metabolism by adiponectin in obesity and diabetes. Biochem Soc Trans 2006;34:798-801.

28 Stefan N, Stumvoll M, Vozarova B, Weyer C, Funahashi T, Matsuzawa Y, Bogardus C, Tataranni PA: Plasma adiponectin and endogenous glucose production in humans. Diabetes Care 2003;26:3315-3319.

29 Combs TP, Berg AH, Obici S, Scherer PE, Rossetti L: Endogenous glucose production is inhibited by the adiposederived protein Acrp30. J Clin Invest 2001;108:1875-1881. 